\title{
THE TOPOLOGICAL UNITARITY IDENTITIES IN CHERN-SIMONS THEORIES
}

\author{
V.Ya.FAINBERG*† and M.S.SHIKAKHWA \\ Department of Physics, Middle East Technical University \\ 06531, Ankara - Turkey
}

October 28,2018

\begin{abstract}
Starting from the generting functional of the theory of relativistic spinors in $2+1$ dimensions interacting through the pure Chern-Simons gauge field,the $\mathrm{S}$-matrix is constructed and seen to be formally the same as that of spinor quantum electrodynamics in $2+1$ dimensions with Feynman diagrams having external photon lines excluded, and with the propagator of the topological Chern-Simons photon substituted for the Maxwell photon propagator.It is shown that the absence of real topological photons in the complete set of vector states of the total Hilbert space leads in a given order of perturbation theory to topological unitarity identities that demand the vanishing of the gauge-invariant sum of the imaginary parts of the Feynman diagrams with a given number of internal on-shell free toplogical photon lines. It is also shown, that these identities can be derived outside the framework of perturbation theory.The identities are verified explicitly for the scattering of a fermion-antifermion pair in one-loop order.
\end{abstract}

*Permanent address: P.N.Lebedev Institute of Physics, Moscow

${ }^{\dagger}$ Work supported in part by the RFFI grant 93-02-3379. 


\section{Introduction}

In the recent years there has been considerable progress in the understanding of theories of charged scalar or spinor particles interacting through the topological ChernSimons (CS) gauge field .It has been shown that the addition of the abelian円CS kinetic term to the Lagrangian of quantum electrodynamics (QED) in $2+1$ dimensions, leads to the appearance of an induced mass of the photon[1].In the work [2], the important symmetry properties of theories with this topological term have been investigated,and some one-loop radiative corrections have been calculatedted. The radiative corrections that the stochastic parameter,i.e the coefficient multiplying the CS term acquires in the framework of perturbation theory have been extensively investigated,and it has been found that for massive matter,one has only finite corrections at one-loop order,and that the two-loop $\beta$-function vanishes[3].Later,a theorem was set which states that there are no corrections to this parameter to all orders of perturbation theory beyond the finite one-loop one [4].

On the non- relativstic domain,it was first proposed by Wilczek that non-relativistic charged particles interacting through the topological CS gauge field can be considered as composite vertices (rigidly bound to a solenoid which is moving along with them) and were named anyons (particles with fractional spin and exotic statistics ). This idea found wide application,and several attempts to apply it to the Fractional Quantum Hall Effect,superfluidity and high temperature superconductivity were made (See the reviews [5] and references therein for details).

In this paper,we are going to consider the theory of relativistic spinor charged particles coupled to the pure topological CS gauge field. This theory has the peculiar property that the propagator and many-particle Green's function of the gauge field are non-zero although real free particles of the gauge field do not exist 2 .

We note that if we consider from the beginning the theory of spinor charged particles

\footnotetext{
${ }^{1}$ In this paper the term CS field will be understood to mean the abelian CS field unless otherwise stated.

${ }^{2}$ The abscence of the topological photons can be seen most generally from the fact that the CS term does not contribute to the Hamiltonian because of its independence of the metric tensor $g_{\mu \nu}$ in curved space-time.
} 
coupled to pure CS gauge field,then the appearance of the parity-conserving radiative corrections to the CS propagator,being finite, does not require the introduction of the Maxwell kinetic counter-term for renormalization. This means that in the framework of perturbation theory,no real photon appears in this theory.This assertion can be proved by starting from a Lagrangian that has in addition to the pure CS term , the Maxwell term;

$$
\frac{1}{4 \gamma} \int\left(F_{\mu \nu} F^{\mu \nu}\right) d^{3} x
$$

If one carries out the calculations of any Feynman diagrams of such a theory and then after renormalization goes to the limit $\gamma \rightarrow \infty$, then one gets the same perturbative results as in the theory with only pure CS term (see also part VI ).

Our main aim in this paper is to show that the absence of the real topological photons in the complete set of vector states of the total Hilbert space of the model,leads to very remarkable topological unitarity identities. These identities demand that in each order of perturbation theory, the gauge-invariant sum of the imaginary parts of all Feynman diagrams with a given number of on-shell internal topological photon lines is equal to zero. Such identities can also be deduced outside the framework of perturbation theory.

In part II we introduce the generating functional of the theory.In part III we construct the S-matrix operator.Part IV demonstrates how to deduce the unitarity identities from the unitarity condition of the theory,and in part $\mathrm{V}$ these identities are verified explicitly for the specific case of the scattering of a fermion-antifermion pair in one-loop order.Part VI is devoted to conclusions and discussion.

\section{The Generating Functional}

We start from the $2+1$ dimensional classical action of spinors coupled to a gauge field whose action is given by the topological pure CS term

$$
\begin{gathered}
S=S_{m}+S_{c s}, \\
S_{m}=\int d^{3} x(\bar{\psi}(i \not \partial+e \not A-m) \psi), \\
S_{c s}=\frac{\mu}{2} \int d^{3} x\left(\varepsilon_{\mu \nu \lambda} A^{\mu} \partial^{\nu} A^{\lambda}\right),
\end{gathered}
$$


Here $\psi$ and $\psi^{\dagger},\left(\bar{\psi}=\psi^{\dagger} \gamma_{0}\right)$ are two dimensional Grassmann spinor fields, and the Dirac matrices in $2+1$ dimensions are taken as [6],

$$
\gamma_{o}=\sigma_{o} \quad, \quad \gamma_{i}=i \sigma_{i} \quad i=1,2
$$

with the $\sigma^{\prime} s$ being the Pauli spin matrices.These $\gamma$-matrices satisfy

$$
\left\{\gamma_{\mu}, \gamma_{\nu}\right\}_{+}=2 g_{\mu \nu} \quad ; \gamma_{\mu} \gamma_{\nu}=g_{\mu \nu}-i \varepsilon_{\mu \nu \lambda} \gamma^{\lambda}
$$

the metric $g_{\mu \nu}$ is defined as

$$
A_{\mu} A^{\mu}=A_{\mu} g^{\mu \nu} A_{\nu} \quad, g_{\mu \nu}=\operatorname{diag} \cdot(1,-1,-1)
$$

$\mu$ and $e$ are two dimensionless coupling constants.A transformation of the gauge field of the form:

$$
A_{\mu} \rightarrow A_{\mu}^{\prime}=\sqrt{\mu} A_{\mu} \quad, \quad \frac{e}{\sqrt{\mu}} \rightarrow g
$$

allows us to have only one coupling constant; $g$ in our theory. Then

$$
S=\int d^{3} x\left(\bar{\psi}(i \not \partial+g \not-m) \psi+\frac{1}{2} \varepsilon_{\mu \nu \lambda} A^{\mu} \partial^{\nu} A^{\lambda}\right)
$$

where we have dropped the prime on $A_{\mu}$. Such a transformation is possible on the quantum level due to the finite renormalization of the stochastic parameter $\mu[3]$.

In order to carry out the path integral quantization of the model,we have to handle the constraints.Noting that we have both first and second class constraints,then generally we have to apply the Batalian-Fradkin-Vilkovisky (BFV) method of quantization[7].However,the triviality of the algebra of the constraints,i.e the fact that the Poisson Brackets of the second class constraints are independent of the fields of the theory,and those of the first class ones are zero,allows us to use the simpler De-Witt-Fadeev -Popov method[8],which will give the same result as that of BFV method[9] 35 The generating functional will then assume the following form

$$
Z\left[\eta, \bar{\eta}, J_{\mu}\right]=(\text { const. }) \int D A_{\mu}(x) D \psi(x) D \bar{\psi}(x) \exp \left\{i S_{m}+i S_{c s}+i S_{g}+i \int d^{3} x\left(\bar{\eta} \psi+\bar{\psi} \eta+J_{\mu} A^{\mu}\right)\right\}
$$

\footnotetext{
${ }^{3}$ The details of this proof will be published in another paper.
} 
$J_{\mu}(\eta, \bar{\eta})$ are external bosonic (fermionic) sources. The action in the above expression differs from the classical one only by the appearence of the gauge-fixing action $S_{g}$;

$$
S_{g}=\int d^{3} x\left(\frac{-1}{2 \alpha}\left(\partial_{\mu} A^{\mu}\right)^{2}\right)
$$

where we have adopted a covariant gauge condition.Integrating Eq.(10) over the gauge field $A_{\mu}$,we get!

$$
\begin{array}{r}
Z\left[\eta, \bar{\eta}, J_{\mu}\right]=\text { const. } \int D \psi D \bar{\psi} \times \exp \left\{\frac{-i}{2} \int d^{3} x d^{3} x^{\prime}\left(I^{\mu}(x) D_{\mu \nu}\left(x-x^{\prime}\right) I^{\nu}\left(x^{\prime}\right)\right)\right. \\
\left.\left.+\int d^{3} x(\bar{\psi}(i \not \partial-m) \psi)+\bar{\eta} \psi+\bar{\psi} \eta\right)\right\},
\end{array}
$$

where

$$
I^{\mu}(x)=J^{\mu}(x)+g \bar{\psi}(x) \gamma^{\mu} \psi(x)
$$

and $D_{\mu \nu}\left(x-x^{\prime}\right)$ is the free CS propagator,

$$
D_{\mu \nu}\left(x-x^{\prime}\right)=\left(-\varepsilon_{\mu \nu \lambda} \partial_{x}^{\lambda}+\frac{\alpha \partial_{\mu} \partial_{\nu}}{\square}\right) \int \frac{d^{3} k}{(2 \pi)^{3}} \frac{e^{i k(x-y)}}{k^{2}+i \epsilon}
$$

Following the general rules,one can determine from the above generating functional all one and many-particle Green's functions of the theory,and use them to construct perturbatively the scattering amplitude for on-shell processes.

Formally,the generating functional (12) is the same as that of QED if one replaces the CS photon propagator by the Maxwell propagator.On the other hand,however,inspite of the existence of nonzero Green's function with two and more external topological photon lines, the corresponding on-shell matrix elements should be zero (!) due to the absence of real topological photons. The consequences of this observation will be investigated in detail in part IV.

\section{The S-matrix Operator}

Although the generating functional Eq.(15) contains all the information of the theory and can be used to derive the scattering amplitudes,it is actually more convenient to

\footnotetext{
${ }^{4}$ We emphasize that strictly speaking, it is necessary to introduce in the exponent of (10) a regularization term,for instance, the Maxwell term Eq.(1), and then take the limit $\gamma \rightarrow \infty$ after integration over $A_{\mu}$.
} 
introduce the S-matrix operator in the theory and use it for this purpose. The S-matrix operator of scalar charged particles coupled to CS gauge field has been constructed in the work [10].In our case we have

$$
\hat{S}=T \exp i S_{\text {int }}\left(\hat{\psi}, \hat{\bar{\psi}}, \hat{A}_{\mu}\right)
$$

where

$$
S_{\text {int }}\left(\hat{\psi}, \hat{\bar{\psi}}, \hat{A}_{\mu}\right)=: \int d^{3} x\left(g \hat{A}^{\mu} \hat{\bar{\psi}} \gamma_{\mu} \hat{\psi}\right):
$$

": :" means normal ordering, and $\hat{\psi}$ and $\hat{\bar{\psi}}$ are now field operators in the interation picture:

$$
\begin{aligned}
& \hat{\psi}(x)=\int \frac{d^{3} p}{(2 \pi)} \sqrt{\frac{m}{E_{\mathbf{p}}}}\left[b^{\dagger}(p) u(p) e^{-i p x}+d^{\dagger}(\mathbf{p}) v(p) e^{i p x}\right] \\
& \hat{\bar{\psi}}(x)=\int \frac{d^{3} p}{(2 \pi)} \sqrt{\frac{m}{E_{\mathbf{p}}}}\left[b^{\dagger}(\mathbf{p}) \bar{u}(p) e^{i p x}+d(\mathbf{p}) \bar{v}(p) e^{-i p x}\right]
\end{aligned}
$$

where $E_{\mathbf{p}}=\sqrt{\mathbf{p}^{\mathbf{2}}+m^{2}}$, and $b(\mathbf{p})(d(\mathbf{p}))$ and $b^{\dagger}(\mathbf{p})\left(d^{\dagger}(\mathbf{p})\right)$ are the annihilation and creation operators of particles(antiparticles) respectively,satisfying the usual anticommutation relations

$$
\left\{b(\mathbf{p}), b^{\dagger}\left(\mathbf{p}^{\prime}\right)\right\}_{+}=\left\{d(\mathbf{p}), d^{\dagger}\left(\mathbf{p}^{\prime}\right)\right\}_{+}=\delta\left(\mathbf{p}-\mathbf{p}^{\prime}\right) .
$$

The orthogonal two-component spinors $u(\mathbf{p})$ and $v(\mathbf{p})$, are respectively the spinors of the positive and negative energy solutions of the free Dirac equation in $2+1$ dimensions, and have the properties:

$$
\begin{aligned}
u(p) \bar{u}(p) & =\frac{\not p+m}{2 m} \\
v(p) \bar{v}(p) & =\frac{\not p-m}{2 m}
\end{aligned}
$$

Obviously,the operatr $\hat{A}_{\mu}(x)$ can not be expanded in terms of the creation and annihilation operators in a manner similar to $\hat{\psi}(x)$ and $\hat{\bar{\psi}}(x)$. We can bring it into use only symbolically with the following property : Only the vacuum expectation value of the product and the T-product of an even number of the operators $\hat{A}_{\mu}$ is nonvanishing,and reduces to the vacuum expectation value of terms with the products and the T-products of two field operators defined as:

$$
\left\langle 0\left|T\left(\hat{A}_{\mu}(x) \hat{A}_{\nu}(y)\right)\right| 0\right\rangle=-i D_{\mu \nu}(x-y)
$$




$$
\begin{aligned}
\left\langle 0\left|\left(\hat{A}_{\mu}(x) \hat{A}_{\nu}(y)\right)\right| 0\right\rangle & =-i D_{\mu \nu}^{\dagger}(x-y) \\
& =-i \int \frac{d^{3} k}{(2 \pi)^{3}}\left(\varepsilon_{\mu \nu \lambda} i k^{\lambda}+\frac{\alpha k_{\mu} k_{\nu}}{\left(k^{2}+i \epsilon\right)}\right) \delta\left(k^{2}\right) \theta\left(k_{0}\right) e^{-i k(x-y)}
\end{aligned}
$$

All the matrix elements of the normal product of any number of the the field operators $\hat{A}_{\mu}(x)$ is equal to zero (by definition !).

We now make a key observation: The expression (16) of the S-matrix is formally identical to that of QED.Therefore,we make the following remarkable statement : All the Feynman diagrams of our theory are identical to those in QED, if we replace the Maxwell photon propagator by the topological CS propogator and exclude all diagrams with on-shell external photon lines.

\section{The Toplogical Unitarity Identities}

Here, we are going to use the S-matrix operator introduced in the previous section to deduce the unitarity identities. However, strictly speaking, we have to consider the S-matrix operator with the counter-terms introduced for the renormalization procedure in each order of perturbation theory(see for example reference [2] for the counter-terms). To avoid additional complications, we will assume that all the matrix elements that we consider have been renormalized.

As we have mentioned above, the absence of the real CS photons means that the complete set of vector states in the total Hilbert space of the system does not contain these topological particles. To investigate the consequences of this fact,we introduce the $\hat{T}$-matrix,

$$
\hat{S}=1-i \hat{T}
$$

where $\hat{S}$ is the S-matrix operator (the energy-momentum conserving $\delta$-function has been suppressed).The unitarity of the S-operator leads to the well-known relation:

$$
i\left(\hat{T}^{\dagger}-\hat{T}\right)=\hat{T} \hat{T}^{\dagger}=2 \operatorname{Im} \hat{T} .
$$

For arbitrary non-diagonal $(|i\rangle \neq|f\rangle)$ matrix elements on mass-shell,we can write the two equivalent relations:

$$
\langle f|2 \operatorname{Im} \hat{T}| i\rangle=\left\langle f\left|\hat{T} \hat{T}^{\dagger}\right| i\right\rangle
$$




$$
\langle f|2 \operatorname{Im} \hat{T}| i\rangle=\sum_{n}\langle f|T| n\rangle\left\langle n\left|T^{\dagger}\right| i\right\rangle .
$$

where in Eq.(26) we have inserted the complete set of physical states $\{|n\rangle\}$ which does not contain the states of the topological photon, but only those of the charged particles. From Eq.(26) we see that in a given order of perturbation theory,the Feynman diagrams that contribute to the imaginary part on the l.h.s can not have internal on-shell topological photon lines because $\{|n\rangle\}$ are physical states. On the other hand,however,investigating Eq.(25) in the framework of perturbation theory shows that diagrams with internal onshell photon lines do appear. This is because the vacuum expectation value of the product of an even number of the symbolic operator $\hat{A}_{\mu}$ does not vanish due to the non-zero value of the imaginary part of the photon propagator(see Eq.(22)). Therefore,demanding the consistency of Eqs.(25) and (26) leads to the important conclusion that in a given order of perturbation theory, the gauge-invariant sum of the imaginary parts of the Feynman diagrams with a given number of on-shell free topological photon lines is equal to zero.The vanishing of this sum of the imaginary parts does not mean the vanishing of the sum of the real parts.As a rule the sum of such Feynman diagrams does not vanish,and will give contribution to the process involved. Each diagram in this sum will be an analytic function of invariant variables. It is also important to underline the fact that,although the gauge-invariant sum of the imaginary parts of the diagrams with a given number of onshell internal photon lines vanishes, the imaginary part of each diagram will not generally vanish. It will vanish only if a diagram is gauge-invariant. This will be demonstrated when we consider a specific example below.

The above arguments give us novel topological unitarity identities which relate the imaginary parts of Feynman diagrams with a given number of internal on-shell free photon lines, whose sum is gauge-invariant. That these identities can be also deduced outside the framework of perturbation theory will be discussed in part VI. 


\section{One-Loop Fermion-Antifermion Scattering}

Now, we illustrate the unitarity identities in the case of scattering of a fermion-antifermion pair in one-loop order.This example is of interest also in the non-relativistic approximation in connection with the perturbative Aharonov- Bohm scattering amplitude.The gaugeinvariant digrams with internal photon lines that contribute to the process are shown in figure 1 below. The analytic expression for the imaginary part of each of these diagrams is:

$$
\begin{array}{r}
A_{a}=\frac{2 g^{4}}{(2 \pi)^{3}} \int d^{3} k d^{3} k^{\prime}\left(\delta^{+}\left(k^{2}\right) \delta^{+}\left(k^{2}\right) \delta\left(p+q-k-k^{\prime}\right) G_{\mu \lambda}(k) G_{\nu \sigma}\left(k^{\prime}\right)\right. \\
\left.\times \frac{\bar{v}(q) \gamma^{\nu}(\not p-\not k+m) \gamma^{\mu} u(p) \bar{u}\left(p^{\prime}\right) \gamma^{\lambda}(p \prime-\not k+m) \gamma^{\sigma} v\left(q^{\prime}\right)}{\left((p-k)^{2}-m^{2}+i \epsilon\right)\left(\left(p^{\prime}-k\right)^{2}-m^{2}+i \epsilon\right)\left(\left(p^{\prime}-k\right)^{2}-m^{2}+i \epsilon\right)}\right), \\
A_{b}=\frac{2 g^{4}}{(2 \pi)^{3}} \int d^{3} k d^{3} k^{\prime}\left(\delta^{+}\left(k^{2}\right) \delta^{+}\left(k^{\prime 2}\right) \delta\left(p+q-k-k^{\prime}\right) G_{\mu \lambda}(k) G_{\nu \sigma}\left(k^{\prime}\right)\right. \\
\left.\quad \times \frac{\bar{v}(q) \gamma^{\nu}(\not k-\not k+m) \gamma^{\mu} u(p) \bar{u}\left(p^{\prime}\right) \gamma^{\sigma}\left(p p^{\prime}-\not k+m\right) \gamma^{\lambda} v\left(q^{\prime}\right)}{\left((k-q)^{2}-m^{2}+i \epsilon\right)\left(\left(p^{\prime}-k\right)^{2}-m^{2}+i \epsilon\right)}\right) .
\end{array}
$$

where $G_{\mu \nu}(k)=\varepsilon_{\mu \nu \alpha} \frac{k^{\alpha}}{\left(k^{2}+i \epsilon\right)}(\alpha=0$ gauge $)$, and $\delta^{+}\left(k^{2}\right)=\delta\left(k^{2}\right) \theta\left(k_{0}\right)$. We for simplicity,restrict ourselves to the case of forward scattering,in which case the imaginary parts of these diagrams give contribution to the total scattering cross-section of the two particles.So,we have now in the center of mass frame for forward scattering (we hereon suppress the irrelevant overall constant):

$$
\begin{array}{r}
A_{a}=\int d^{3} k d^{3} k^{\prime}\left(\delta^{+}\left(k^{2}\right) \delta^{+}\left(k^{\prime 2}\right) \delta\left(p+q-k-k^{\prime}\right) G_{\mu \lambda}(k) G_{\nu \sigma}\left(k^{\prime}\right)\right. \\
\left.\times \frac{\left(\gamma^{\nu}(\not p-\not k+m) \gamma^{\mu}\right)_{q p}\left(\gamma^{\lambda}(\not p-\not k+m) \gamma^{\sigma}\right)_{p q}}{4(p . k)^{2}}\right) \\
A_{b}=\int d^{3} k d^{3} k^{\prime}\left(\delta^{+}\left(k^{2}\right) \delta^{+}\left(k^{\prime 2}\right) \delta\left(p+q-k-k^{\prime}\right) G_{\mu \lambda}(k) G_{\nu \sigma}\left(k^{\prime}\right)\right. \\
\left.\times \frac{\left(\gamma^{\nu}(\not p-\not k+m) \gamma^{\mu}\right)_{q p}\left(\gamma^{\sigma}\left(\not p-\not k^{\prime}+m\right) \gamma^{\lambda}\right)_{p q}}{4(p . k)\left(p . k^{\prime}\right)}\right) .
\end{array}
$$

where $(\ldots)_{q p}=\bar{v}(q)(\ldots) u(p)$. Noting the symmetry of the integrand in $\mathrm{k}$ and $k^{\prime}$, using the identities (20), and taking traces over the $\gamma$-matrices as we proceed,we get after somewhat lengthy calculations :

$$
A_{a}=-\int d^{3} k \delta^{+}\left(k^{2}\right)\left(1+\frac{p \cdot k}{m^{2}}+\frac{q \cdot k}{p . k}\right)
$$




$$
A_{b}=\int d^{3} k \delta^{+}\left(k^{2}\right)\left(1+\frac{p \cdot k}{m^{2}}+\frac{q \cdot k}{p \cdot k}\right) .
$$

Then $A_{a}=-A_{b}$, or;

$$
A_{a}+A_{b}=0
$$

The same result can be obtained for non-forward scattering toof.This result verifies the unitarity identities in one-loop oder for the scattering of a fermion-antifermion pair.

\section{Conclusions and Discussion}

In conclusion, we first note that the fact that the topological unitarity identities do not require the vanishing of the imaginary part of each Feynman diagram with internal on-shell photon lines,can be easily understood for the diagrams in figure 1.First of all,each of these diagrams is not gauge-invariant by itself,only their sum is.Moreover, the three diagrams that are the variations of the diagram in figure 1.a, are the different boundary values of a single analytical function of the two invariant variables $s=(p+q)^{2}$ and $t=\left(p-p^{\prime}\right)^{2}$ for different values of these variables(i.e in different channels).

The topological unitarity identities give additional possibility to check the self-consistency of the Chern-Simons theories, and to simplify the calculation of Feynman diagrams in perturbative analysis of the theory; in particular the coefficients multiplying these diagrams.They also provide additional criteria for correctness and gauge- invariance of the diagrams that resemble the Ward identities.

We have carried out some analogous calculations in the theory of scalar charged particles interacting through pure CS gauge field.In this case there are four one-loop diagrams with two internal photon lines that contribute to the scattering amplitude of two opposittely charged particles. The sum of the imaginary parts of these diagrams (which is gaugeinvariant) vanishes too. We also note that the generalization to the non-abelian case should not be difficult.

It is necessary to undeline that the S-matrix operator of the theory of charged particles interacting through the CS gauge field (see for example Eq. (15)) contains non-zero

\footnotetext{
${ }^{5}$ The calcualtions for non-forward scattering will be reported in another work.
} 
normal-ordered products terms of the on-shell topological photon operators. However, all the matrix elements of the normal-ordered products of the topological photon operator are "by definition" equal to zero. Due to this property, the matrix elements $\left\langle f\left|T^{\dagger} T\right| i\right\rangle$ in Eq.(25) contain terms with internal topological $D_{\mu \nu}^{+}(x)$ functions (See Eq.(30)). Thus, a comparative analysis of Eqs.(25) and (26) non-perturbatively shows that the only difference between them comes from these terms. So, we come to the conclusion that these two equations will be consistent only in the case when each exact matrix element with a given number of internal topological lines in Eq.(25) vanishes. This statement is a direct generalization of the topological unitarity identities in CS theories to the non-perturbative case.

Moreover, the same arguments are also applicable in the non-relativistic case, and analogous identities can be deduced there too.

Finally, we note that if we introduce into the action in (10) the Maxwell term (1), then we get (We do not perform the transformation (7) here) formally the same generating functional Eq.(12) except that the $D_{\mu \nu}$ propagator is replaced by (in momentum space)

$$
D_{\mu \nu}(p)=\gamma\left(\frac{g_{\mu \nu}-p_{\mu} p_{\nu} / p^{2}}{p^{2}-\mu^{2} \gamma^{2}+i \epsilon}+\frac{i \mu \gamma \varepsilon_{\mu \nu \lambda} p^{\lambda}}{\left(p^{2}+i \epsilon\right)\left(p^{2}-\mu^{2} \gamma^{2}+i \epsilon\right)}\right)+\alpha \frac{p_{\mu} p_{\nu}}{\left(p^{2}+i \epsilon\right)^{2}}
$$

In the above propagator, the photon acquires the mass $\mu \gamma$ as we have mentioned in the introduction. The Maxwell term also plays the role of a regularization term and the theory becomes superrenormalizable [6]. Since the stochastic parameter $\mu$ and the charge $e$ acquire only finite corrections due to the superrenormalizability of the theory, then it is possible to consider two limiting procedures (in each order of perturbation theory after renormalization). In the case $\gamma \rightarrow \infty$ the propagator (34) reduces to the pure CS propagator, and we get a renormalizable theory. Thus, in this case the topological unitarity identities hold. In the case $\mu \rightarrow 0, \gamma$ finite, we obtain pure $2+1$ dimensional QED propagator, and the unitarity identities no longer hold. In the intermediate cases, one can show that the total operator of the gauge field in the S-matrix consists of two parts: the operator of the real massive photon, and that of the massless topological photon that does not give real radiation. Thus, the topological unitarity identities still hold in the sense that the gauge-invariant sum of the imaginary parts of the Feynman diagrams with at 
least one on-shell internal topological line vanishes.

\section{Acknowledgments.}

We thank Professor N.K.Pak for helpful discussions and M.Boz who also checked the vanishing of the sum of the imaginary parts in the scalar case. One of us (V.F) thanks the directorate of ICTP where this work was concluded for hospitality.

\section{References}

[1] R.Jackiw and S.Templeton, Phys.Rev.D 23,2291 (1981); J.Schonfeld, Nucl.Phys.B

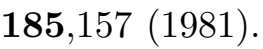

[2] S.Deser,R.Jackiw and S.Templeton, Ann.Phys.(N.Y) 140,372 (1982).

[3] G.W.Semenoff,P.Sodano and Y-S.Wu, Phys.Rev.Lett.62,715 (1989); W.Chen, Phys.Lett.B 251,415 (1990); D.K.Hong,T.Lee and S.H.Park, Phys.Rev.D 48,3918 (1993).

[4] S.Coleman and B.Hill, Phys.Lett.B 159,184 (1985).

[5] F.Wilczek, Fractional Statisties And Anyon Superconductivity, (World Scientific,Singapore 1990), R.Prange and S.Girvin (eds.), The Quantum Hall Effect, (Springer,Berlin 1986).

[6] G.Scharf,W.F.Wreszinski,B.M.Pimentel and J.L.Tomazelli, Ann.Phys. (N.Y) 231,185 (1994).

[7] E.S.Fradkin and G.A.Vilkovisky, Phys.Lett.B 55,224 (1975); J.A Batalin and G.A.Vilkovisky, Phys.Lett.B 69,309 (1977); E.S.Fradkin and T.E.Fradkina, Phys.Lett.B 72,343 (1978).

[8] B.S.de Witt, Phys.Rev.162, 1195 (1967); L.D.Fadeev and V.N.Popov, Phys.Lett.B 25,30 (1967);

[9] V.Ya.Fainberg and N.K.Pak, Journ.Theor.Math.Phys.103, 328 (1995). 
[10] M.Boz,V.Ya.Fainberg and N.K.Pak, Phys.Lett.A207, 1(1995). 